\title{
Philosophiques
}

\section{L’intentionnalité, naturellement!}

\section{Luc Faucher}

Volume 34, numéro 1, printemps 2007

Cosmopolitisme et particularisme

URI : https://id.erudit.org/iderudit/015870ar

DOI : https://doi.org/10.7202/015870ar

Aller au sommaire du numéro

Éditeur(s)

Société de philosophie du Québec

ISSN

0316-2923 (imprimé)

1492-1391 (numérique)

Découvrir la revue

Citer ce document

Faucher, L. (2007). L'intentionnalité, naturellement! Philosophiques, 34(1),

165-171. https://doi.org/10.7202/015870ar d'utilisation que vous pouvez consulter en ligne.

https://apropos.erudit.org/fr/usagers/politique-dutilisation/ 


\title{
L'intentionnalité, naturellement !
}

\author{
LUC FAUCHER \\ Université du Québec à Montréal \\ Faucher.luc@uqam.ca
}

Les prétentions de Pierre Jacob dans ce livre ne sont pas de développer une thèse positive concernant l'intentionnalité (pour cela on consultera plutôt son autre ouvrage Pourquoi les choses ont-elles un sens ?), mais plutôt de présenter une géographie détaillée du traitement philosophique de la question de l'intentionnalité au vingtième siècle. L'auteur s'acquitte admirablement de sa tâche, avec pour résultat que le livre devra maintenant figurer en bonne place dans les bibliographies des cours de philosophie de l'esprit. Cela ne rend pas mon travail de commentateur très facile, mais, en cherchant bien, deux passages me semblent faire problème.

1. Tout d'abord, dans sa discussion de la position des Churchland concernant l'intentionnalité, Jacob écrit la chose suivante:

Selon le matérialisme éliminatif, c'est une erreur de croire que les êtres humains ont des intentions, des désirs et des croyances. C'est donc une erreur de croire que les croyances existent. Si la croyance que les croyances existent est elle-même fausse, alors la croyance que les croyances n'existent pas est vraie. Mais si cette croyance était vraie, alors elle serait fausse, puisqu'il existerait au moins une croyance. À moins de renoncer à la distinction entre les croyances vraies et les croyances fausses, il est paradoxal de croire que les croyances n'existent pas " (Jacob, 2004, 163).

Cet argument, qui revient parfois dans la littérature, ne m'a jamais convaincu. L'éliminativiste des croyances est bien sûr dans une situation particulière comparativement aux autres éliminativistes. Un éliminativiste des races peut sans apparence de paradoxe faire des énoncés du type «il ne faut pas croire que "les races" existent » pour indiquer que le concept "race” n'a tout simplement pas de référent. Le "problème" des éliminativistes des croyances est qu'ils doivent utiliser dans leur injonction le concept même qu'ils souhaitent éliminer ${ }^{1}$ : par exemple, “il ne faut pas avoir la croyance que les 'croyances' existent”. Pour comprendre pourquoi nous n'avons pas affaire à une formule paradoxale (et pourquoi l'éliminativisme n'est pas affecté par ce genre d'argument), il faut replacer l'injonction dans le cadre de la théorie réductionniste que défendent les Churchland. Pour un certain nombre de raisons qu'il serait

1. En fait, comme me l'a fait remarquer Pierre Poirier, la situation de l'éliminativiste des croyances pourrait finalement ressembler à celle de l'éliminativiste des races. Selon lui, Churchland pourrait dire non pas qu'il «ne croit pas que les croyances existent », mais bien plutôt, qu'il « dit que les croyances n'existent pas", et ces paroles sont causées par un état de mon cerveau. Churchland pourrait alors considérer l'argument de Jacob comme une pétition de principe contre sa position. 
un peu long d'expliquer, le modèle de la réduction classique comme Nagel l'a formulé a été abandonné. Un des problèmes qui a mené à cet abandon est la difficulté à établir des lois-pont entre concepts de théories de niveaux différents lorsque le concept de niveau supérieur s'avère n'avoir pas de référent ou bien lorsqu'il doit être corrigé substantiellement. La nouvelle version du réductionnisme défendue par les Churchland (que Bickle [1998] nomme le réductionnisme nouvelle-vague) propose une vision différente des relations interthéoriques : dans le cas d'une réduction, une image corrigée ou analogue à la théorie de haut niveau est construite à partir des ressources conceptuelles fournies par la théorie de bas niveau. Cet analogue reflète dans une certaine mesure les propriétés formelles et structurelles de la théorie de haut niveau. Il peut être déduit de la théorie de bas niveau (c'est donc un cas de réduction inter-théorique), mais les relations entre l'analogue et la théorie de haut niveau sont des relations analogiques, c'est donc dire qu'elles peuvent varier sur un continuum allant de «fortement analogue » à "faiblement analogue ». Dans le cas où le concept de bas niveau est «faiblement analogue » au concept de haut niveau, ce dernier peut-être soit révisé radicalement soit éliminé; alors que dans le cas où le concept de bas niveau est "fortement analogue » au concept de haut niveau, ce dernier peut être dit réduit au premier. Revenons maintenant au cas des croyances.

Ce qu'affirment les Churchland, c'est que, au vu du développement actuel (ou futur) des neurosciences, le concept ordinaire de croyance est (ou sera probablement) un concept inadéquat (ou insuffisant) pour expliquer la cognition humaine (voir Churchland et Sejnowski, 1989 et 1990, p. 232). Dire que le concept de croyance est inadéquat (ou insuffisant) n'équivaut cependant pas à dire qu'aucun autre concept ayant un profil fonctionnel similaire ne prendra la place. La théorie de la réduction défendue par Churchland postule en effet que l'analogue neurobiologique reflètera dans une certaine mesure les propriétés formelles ou structurelles des éléments de haut niveau. Le concept neurobiologique qui remplacera celui de croyances aura, on peut le souhaiter, la capacité d'expliquer tout ce qu'expliquait le concept de croyance, sans en avoir les limitations (par exemple, l'incapacité à expliquer les propriétés des états représentationnels infra-linguistiques exhibées par les réseaux de neurones). Affirmer que les croyances n'existent pas ou qu'elles doivent être éliminées ne conduit donc pas à un paradoxe puisqu'on espère qu'un concept $\mathrm{X}$ plus adéquat, dans une théorie neurobiologique, permettra de rendre compte de ce que nous nommons actuellement les croyances (sans avoir nécessairement toutes les propriétés que nous attribuons aux croyances). C'est à ce genre de quasi-équivalent fonctionnel que font allusion Churchland et Sejnowski lorsqu'ils écrivent :

Plutôt que de partir du vieux modèle logico-phrastique, nous modélisons le traitement de l'information comme la trajectoire d'un système complexe non linéaire dans un espace à plusieurs dimensions. Cette structure ne ressemble pas à des phrases déployées en séquences logiques, mais elle est potentiellement assez riche 
et complexe pour donner lieu à des comportements capables de soutenir des relations logiques et sémantiques (1989 et 1990, p. 234).

Ce concept X pourra prendre la place du concept de «croyance» dans les énoncés du genre «Je crois que les croyances n'existent pas » de telle façon que nous aurons des énoncés du type «Je X que les croyances n'existent pas ». Ainsi, Paul Churchland peut-il souhaiter que, dans un avenir rapproché, "notre compréhension mutuelle et même notre introspection pourraient [...] être reconstituées à l'intérieur du cadre des neurosciences complétées, une théorie dont nous espérons qu'elle sera beaucoup plus puissante que la théorie du sens commun qu'elle remplace [...]» (1981-1990, p. 206).

En fait, malgré leur éliminativisme, et cela permet de mieux comprendre ce dont ils se font les avocats, les Churchland présentent une variante du programme de naturalisation de l'intentionnalité ; leur traitement de la question est à certains égards assez semblable à celui de Drestke ou de Millikan. Dans «Stalking the Wild Epistemic Engine» (1983 et 1990), par exemple, ceux-ci argumentent en faveur de ce qu'ils nomment un programme "naturaliste " qu'ils opposent au «rationalisme» du paradigme computationnel classique en sciences cognitives, dont les représentants principaux sont Fodor et Pylyshyn). Un élément important de ce programme est le fait que nous devons nous considérer (tout comme les autres produits de la sélection naturelle) comme des "engins épistémiques » (un autre élément est le rejet du paradigme propositionnel qui affirme que les états représentationnels sont des attitudes propositionnelles et que les relations entre ces états sont fonction des relations logiques entre les contenus de ces états $\left.{ }^{2}\right)$. Est un engin épistémique:

[...] tout mécanisme qui exploite le flux d'énergie environnementale, et l'information qu'il contient, pour produire plus d'information, et pour guider le mouvement. Pour autant que les engins épistémiques naturels (ou sauvages) sont concernés, la survie dépend de l'adéquation entre l'information qu'ils contiennent et le monde qu'ils habitent ${ }^{3}$ (1983 et 1990, p. 302).

Le problème est donc d'expliquer comment les organismes en viennent à être calibrés avec leur environnement. Dans certains cas, nous expliquent Drestke et Millikan, l'évolution se charge de la calibration; dans d'autres, la calibration est établie par l'apprentissage qui conduit à modifier les états neuronaux pour qu'ils varient de façon fiable avec les états du monde. Leur définition du contenu calibrationnel est la suivante:

2. «Dans la conception connexionniste, les représentations s'avèrent être des patterns d'activation à travers un ensemble d'unités; d'un point de vue mathématique, ce sont des vecteurs dans un état spatial. Les computations se révèlent être des transformations de vecteurs d'un état spatial à un autre» (Patricia Churchland, 1991, p. 22).

3. La traduction est de moi. 
Un état $\mathrm{E}$ d'un système $\mathrm{O}$ a le contenu calibrationnel $\mathrm{P}$ si et seulement si $\mathrm{O}$ ne serait pas en E sans que $P$, avec un haut degré de probabilité n/m (1983 et 1990, p. 308$)^{4}$.

Ce contenu calibrationnel peut être produit par une corrélation entre un état du monde et un état d'un système sensoriel assez périphérique, ou bien entre un état du monde et un système moins périphérique (c'est-à-dire qui intègre l'information de plusieurs systèmes périphériques). Selon Drestke et Millikan, chaque système pourrait avoir une structure représentationnelle particulière (lui permettant par exemple d'exploiter certaines régularités physiques différentes). Quelques-uns de ces systèmes utiliseront peut-être des représentations semblables au langage, mais, nous préviennent les Churchland, ces structures pourraient jouer un rôle mineur dans la cognition humaine.

La majeure partie de la cognition pourrait prendre place dans d'autres soussystèmes, et suivre des principes inapplicables au domaine linguistique ${ }^{5}$ (1983 et 1990, p. 309).

Ainsi, on le voit, l'éliminativisme concernant les croyances n'est pas un obstacle au projet de naturalisation de l'intentionnalité ; au contraire, il n'est qu'une version (radicale, si on veut) de ce projet où le centre d'intérêt n'est plus (uniquement) la naturalisation d'états comme les croyances, mais plutôt celle d'entités non propositionnelles et non linguistiques.

2. Ma seconde remarque porte sur une critique adressée à la version de Millikan du programme de naturalisation de l'intentionnalité. Comme l'écrit Jacob: "Selon l'hypothèse consumériste [de Millikan], le contenu de la représentation est fixé par ce que le mécanisme consommateur $M^{*}$ a besoin de "savoir" pour remplir sa fonction dans des conditions normales " (193). Cela entraînerait le problème suivant :

Mais si le contenu d'une représentation dépendait aussi étroitement de la fonction propre du mécanisme consommateur que le soutient Millikan, alors le système visuel d'une mouche mâle ne devrait pas seulement lui permettre de discriminer une mouche femelle d'un caillou, d'un oiseau et d'un avion. Il devrait aussi lui permettre de discriminer une mouche femelle saine d'une mouche femelle contaminée par un virus mortel. Il devrait lui permettre de discriminer une mouche femelle fertile d'une mouche femelle stérile. [...] N'est-ce pas surcharger les capacités de discrimination du système visuel des mouches mâles que d'exiger qu'il transmette au système consommateur une représentation dotée d'un tel contenu (195).

Mon objection à ce genre de remarque est la suivante : c'est une question empirique que de savoir ce que les mouches mâles peuvent discriminer et ce dont elles ont besoin pour survivre. On ne peut, à mon avis, sur la seule

4. Idem.

5. Idem. 
base de ce qui serait adaptatif pour lui, décider de ce qu'est ou devrait être le contenu des représentations d'un organisme sans risquer de faire des erreurs du type de celle commise par les premiers critiques de la théorie de la sélection parentèle (kinship selection), selon laquelle les fourmis n'ont pas les capacités intellectuelles ou perceptives suffisantes pour effectuer les calculs complexes nécessaires déterminant pour qui elles doivent se sacrifier. Après tout, Mère Nature est ingénieuse, elle peut trouver des solutions économiques à ce genre de problème (par exemple, Mère Nature pourrait doter les mouches mâles d'un gabarit de mouche femelle qui serait tel que les premières ne seraient attirées que par les mouches fertiles et en bonne santé. Cette solution ressemble à celle que les psychologues évolutionnistes croient que Mère Nature utilise pour nous. Selon eux, nous serions attirés par certaines qualités corrélées avec la fertilité (le grain de la peau) et avec la santé (la symétrie) ${ }^{6}$. Si le monde ne coopère pas ou si l'ingénuité de Mère Nature n'est pas suffisante, c'est-à-dire s'il n'existe pas d'indices qui co-varient avec les propriétés que l'organisme aurait avantage à détecter, ou si Mère Nature n'a pas doté l'organisme de la capacité (ou même de la disposition à acquérir cette capacité) à détecter ces indices, le système ne pourra consommer cette information puisqu'elle n'existe tout simplement pas -- ou du moins, elle n'existe pas pour lui --, et on ne pourra pas attribuer ce contenu à ses états internes.

Ces quelques remarques concernant l'usage tous azimuts de ce qu'on nomme parfois l'" adaptationnisme logique " (l'utilisation de l'adaptationnisme comme heuristique pour l'attribution de contenu et de fonction), me semblent indiquer les limites à l'intérieur desquelles son usage est légitime. Il est clair qu'à lui seul l'adaptationnisme logique ne permet pas d'établir ou de fixer le contenu des représentations. En fait, pour reprendre une thèse défendue par Brian Keeley (1999), si l'on regarde la façon dont est établi le contenu et les fonctions dans le champ de la «neuroethologie» (pour prendre un domaine de la science dans lequel la fixation des croyances est une question centrale; voir Keeley, 2000, pour une description de ce champ), il s'avère que la fixation des contenus et des fonctions demande plus que l'utilisation de la simple pensée adaptationniste. Selon lui, pour établir une fonction ou un contenu, «[...] on a besoin des données concurrentes provenant de l'analyse béhaviorale, de l'analyse anatomique, de la physiologie, de l'éthologie, de la biologie évolutionniste et du raisonnement adaptationniste ». Par exemple, comme je le remarquais plus haut, une contrainte sur ce qui peut figurer dans le contenu des représentations est l'étendue du domaine de réception des organes sensoriels. Un animal ne peut répondre à ou consommer un type de stimulus que si son système sensoriel est en mesure de le détecter.

6. Mieux encore, Downes (2001) et Keeley (2002) notent qu'en fait la détection de ces indices particuliers pourraient être inutiles. Ils citent à cet effet les travaux de Kohl et ceux de Halpern qui montrent que l'information concernant la fertilité pourrait bien plutôt être conviée par l'odorat ou par ce qu'on nomme l'organe voméronasal dont la lésion chez l'humain provoque une baisse importante des comportements reproductifs. 
Cette façon de concevoir le problème de la fixation du contenu des représentations ouvre la porte à une série de questions concernant les sens qui n'ont pas reçu, je crois, toute l'attention qu'elles méritent dans la littérature philosophique. La première de ces questions concerne la façon de distinguer les systèmes sensoriels et de leur assigner un domaine. Keeley, dans un texte récent (2002), propose quatre critères qui, pris ensemble, sont nécessaires et suffisants pour identifier une modalité sensorielle. Parmi ceux-ci, la nécessité d'identifier l'usage ou la fonction propre du sens (dedication). Cette question est importante puisqu'un organe peut détecter une forme d'énergie sans être conçu pour ce faire. Aussi doit-on distinguer entre ce que les éthologues nomment « détection» et « réception». Par exemple, quoique nous n'ayons pas de modalité pour l'électroréception, nous sommes capables d'électrodétection, c'est ce qui se produit quand on place sa langue sur une pile de neuf volts chargée. Dans ce cas, ce sont nos récepteurs de goût, de douleur, ainsi que nos récepteurs tactiles qui nous permettent de détecter l'électricité. Une autre question est celle soulevée par Kathleen Akins, et elle concerne le narcissisme possible des représentations. Selon elle, ceux qui veulent naturaliser l'intentionnalité commettent l'erreur de croire qu'une des fonctions des sens est de représenter le monde de façon véridique. Utilisant l'exemple de la thermoréception, Akins montre que le rôle de cette modalité n'est pas d'indiquer une propriété statique (la température dans le monde), mais bien plutôt une propriété dynamique (comment la chaleur ou le froid m'affecte-t-il ?). L'information conviée par le système de thermoréception dépend de mes besoins thermaux spécifiques, besoins qui sont tributaires entre autres de ma physiologie, de mon environnement adaptatif, etc. Akins propose en fait une théorie dont je crois qu'elle aura l'heur de plaire à Jacob, mais qui pose un problème à la plupart des versions du programme naturaliste. Dans le cas des systèmes sensoriels, il y a tout lieu de croire qu'il existe une relation "symbiotique » entre l'information amassée par le système et l'information nécessaire au système moteur. Si tel est le cas, étant donné que les sens ne représentent pas le monde (out there), mais qu'ils n'ont pour but que de permettre la survie par le biais d'actions adaptées, la stratégie naturaliste classique qui consiste à tenter de comprendre l'intentionnalité de haut niveau à partir de l'intentionnalité de plus bas niveau des sens semble avoir un grave problème. Le problème devant lequel se trouvent les naturalistes devient donc celui de comprendre la raison et la modalité du passage de ce que Akins nomme le " projet sensorimoteur " (encoder l'information nécessaire pour le mouvement) au "projet ontologique» du cerveau (nous donner un monde d'objets et de propriétés stables et persistants).

Une façon de résumer le contenu de mes remarques aux deux passages du livre de Jacob serait de dire la chose suivante: étant donné que l'intentionnalité des états mentaux est un phénomène naturel, il convient, pour l'étudier, de se tourner sérieusement vers les sciences naturelles (principalement la biologie et la psychologie, mais aussi l'éthologie cognitive). Je ne veux pas 
dire par là qu'il faut que la philosophie abdique tout rôle dans la discussion des questions reliées à l'intentionnalité, mais simplement qu'elle évite le plus possible la philosophie de salon (armchair philosophy) et qu'elle soit donc informée par les résultats venant des domaines pertinents aux questions qu'elle traite. Je ne crois pas que Jacob soit un philosophe de salon, loin de là, mais mes réponses à ses deux objections montrent à mon avis que l'adoption d'une posture véritablement (ou encore plus) naturaliste a le potentiel de modifier considérablement la forme et le contenu des questions philosophiques sur l'intentionnalité. Si je n'avais pas été habitué à un certain scepticisme concernant la notion de "progrès » en philosophie, je dirais même qu'un naturalisme comme celui auquel je fais référence pourrait faire progresser les discussions sur l'intentionnalité".

\section{Bibliographie}

Akins, K. "Of Sensory Systems and the 'Aboutness' of Mental States », Journal of Philosophy, 337-372, 1996.

Churchland, P. S. «Les neurosciences concernent-elles la philosophie ? », in J. M. Missa (dir. publ.), Philosophie de l'esprit et sciences du cerveau, Paris, Vrin, p. 11-22, 1991.

Churchland, P. et P. S. Churchland. «Stalking the Wild Epistemic Engine", in W. G. Lycan (dir. publ.), Mind and Cognition: A Reader, Cambridge, Blackwell, p. 300-311, 1983 et 1990.

Churchland, P.S. et T. J. Sejnowski. «Neural Representation and Neural Computation », in W. G. Lycan (dir. publ.), Mind and Cognition: A Reader, Cambridge, Blackwell, p. 224-252, 1989 et 1990.

Downes, S. M. "Some Recent Developments in Evolutionary Approaches to the Study of Human Cognition and Behavior ", Biology and Philosophy, 16, p. 575595, 2001.

Jacob, P. Pourquoi les choses ont-elles un sens? Paris, Odile Jacob, 1997.

—. L'intentionnalité: problèmes en philosophie de l'esprit, Paris, Odile Jacob, 2004.

Keeley, B. «Fixing Content and Function in Neurobiological Systems: The Neuroethology of Electroperception ", Biology and Philosophy, 14, p. 395-430, 1999.

- - "Neuroethology and the Philosophy of Cognitive Science ", Philosophy of Science, vol. 67, $\mathrm{n}^{\circ}$ 3, S404-S417, 2000.

—. "Making Sense of the Senses: Individuating Modalities in Humans and Other Animals ", Journal of Philosophy, vol. XCIX, n 1, p. 5-28, 2002.

* Je remercie Pierre Poirier pour ses remarques toujours pertinentes sur une version précédente de ce commentaire. 